\title{
Double Implementation in a Market for Indivisible Goods with a Price Constraint
}

\author{
Helmuts Āzacis * \\ Departament d'Economia i d'Història Econòmica \\ Universitat Autònoma de Barcelona
}

September 10, 2004

\begin{abstract}
I consider the problem of assigning agents to objects where each agent must pay the price of the object he gets and prices must sum to a given number. The objective is to select an assignment-price pair that is envyfree with respect to the true preferences. I prove that the proposed mechanism will implement both in Nash and strong Nash the set of envy-free allocations. The distinguishing feature of the mechanism is that it treats the announced preferences as the true ones and selects an envy-free allocation with respect to the announced preferences.

Journal of Economic Literature classification numbers: C78, C71, D78

Keywords: Indivisible Goods, Envy-Freeness, Implementation, Strong Nash Equilibrium
\end{abstract}

*I am grateful to Jordi Massó for detailed and time-consuming checking of initial drafts, and all other help. I would also like to thank Carmen Beviá, David Pérez-Castrillo and Flip Klijn for helpful comments. This research was undertaken with support from the European Community's Phare ACE Programme 1998 (contract number P98-2007-S). The content of the publication is the sole responsibility of the author and it in no way represents the views of the Commission or its services. Financial support from the Spanish Ministry of Science and Technology through grant BEC2002-02130 is gratefully acknowledged. My email: hazacis@idea.uab.es 


\section{Introduction}

I study the problem of assigning a set of indivisible objects to a set of agents. Each agent wants exactly one object, and his preferences are quasilinear in money. Each agent must pay a price corresponding to the object he gets, and prices are required to sum to a given number. A standard example is the housemate problem where a group of tenants is sharing an apartment. The objective is to determine who gets which room and how much he must pay subject to the constraint that the sum of their contributions must equal the rent of the apartment. Alternatively, besides the indivisible objects there is a divisible object - money - that must also be shared among agents, for example, heirs sharing inheritance. In this case at least some prices will be negative. Nor the valuations of objects are required to be positive, that is, the objects need not necessarily be goods, but can also be bads or burdens. For example, the central government with fixed budget is assigning waste disposal sites and other duties/projects to municipalities.

When deciding on the assignment of agents to objects and the corresponding prices, we may want to meet certain criteria. The usual requirements include efficiency, since it maximizes the welfare of the society; envy-freeness (each agent prefers the object he has been assigned to any other object), since it is a sufficient condition for the stability of the assignment; and individual rationality (agents never get an utility lower than the one they would obtain if they exercise their outside options), since it assures that all agents would like to participate. Besides normative criteria that we would like to meet there can be other restrictions imposed. For example, it is reasonable to require that in the room-sharing problem prices are nonnegative. Usually all above-mentioned criteria cannot be met simultaneously. For example, in the housemate problem if the rent is too high, individual rationality will be violated, and additionally there usually exists a trade-off between envy-freeness and nonnegativity of prices.

There exists wide literature that uses the above framework. Each contribution usually provides an algorithm designed to select a particular allocation with some desirable properties. For example, algorithms by Abdulkadiroğlu et al. [1] and Haake et al. [8] select envy-free allocations while Brams and Kilgour [5] and Chin Sung and Vlach [6] impose nonnegative prices. This paper adopts envy-freeness as a solution concept while ignoring individual rationality and signs of prices. Individual rationality depends on the price constraint - that the prices must sum to a given number - something that is given exogenously in the model. And since the model does not impose restrictions on the signs of neither valuations of objects nor the price constraint, so I do not impose restrictions on the signs of prices. Additionally, envy-freeness implies efficiency therefore envy-freeness is considered such an attractive solution concept.

All cited algorithms take valuations of objects as given and ignore strategic incentives of agents to misreport their true preferences to achieve higher utility. Thus the properties of allocations selected by different algorithms with respect 
to the true but unknown preferences can be quite different from the properties with respect to the announced preferences. Therefore, if we insist on using an algorithm to propose a particular allocation, two related questions arise: (1) are all (strong) Nash equilibrium outcomes of the game induced by the algorithm envy-free? and (2) are all envy-free allocations outcomes of some (strong) Nash equilibrium of the game? That is, does the algorithm (strong) Nash implement the set of envy-free allocations?

I propose the mechanism where agents announce their valuations, not necessarily the true ones, and an allocation is selected that is envy-free with respect to the announced preferences. I prove that, although truth-telling is not an equilibrium strategy, in any (strong) Nash equilibrium the reported preferences will be such that agents will be envy-free with respect to the true preferences. The allocation that the mechanism selects, given the announced preferences, coincides with the one that would be selected according to the algorithm of Abdulkadiroğlu et al. [1]. Therefore the paper provides the justification for the use of the algorithm on strategic grounds. The advantage of this particular algorithm is that it provides a formula for the selected price vector in terms of the announced preferences, making it easy to verify existence of profitable deviations. On other hand, since the algorithm by Abdulkadiroğlu et al. [1] does not specify which efficient assignment to select with respect to the announced preferences, I have introduced a tie-breaking rule to ensure that the assignment is efficient with respect to the true preferences.

Papers related to the present work are by Tadenuma and Thomson [14] and Beviá [4]. The former work considers allocating only one indivisible object to one of several agents when monetary compensations are available while the later extends it to several objects and agents. Both consider direct revelation games that select envy-free allocations, given announced preferences, and ask for the scope to manipulation. Both prove that the set of equilibrium outcomes coincides with the set of envy-free allocations with respect to the true preferences.

The remaining of the paper is organized as follows. The following section provides the formal model and some results necessary for the proof. Section 3 defines the implementation problem and states the theorem. Before proving the theorem in Section 5, an example for two-agent two-object case is provided in Section 4. Final remarks in Section 6 conclude the paper. Some of the proofs are relegated to the Appendix.

\section{Preliminaries}

The set of agents is $I=\{1, \ldots, n\}$ and the generic elements of $I$ will be denoted by $i$ and $k$. The set of objects is $J=\{1, \ldots, n\}$ with generic elements of $J$ denoted by $j$ and $l$. Throughout it is assumed that the number of agents and 
objects is the same $n .{ }^{1}$ It is assumed that each agent wants one and only one object. The assignment of agents to objects is given by a one-to-one mapping $\mu: I \rightarrow J$. The price vector is $p=\left(p_{1}, \ldots, p_{n}\right) \in R^{n}$. The matrix of true valuations is $A=\left[a_{i j}\right]_{i \in I, j \in J}$ where $a_{i j} \in R$ is the valuation that agent $i$ assigns to object $j$. Utilities are quasi-linear in prices, namely, the utility of agent $i$ from being assigned to object $\mu(i)$ and paying its price $p_{\mu(i)}$ is $u_{i}\left(p_{\mu(i)}\right)=a_{i \mu(i)}-p_{\mu(i)}$. Let $M$ denote the set of assignments. An allocation is an assignment-price pair $(\mu, p) \in M \times R^{n}$.

Definition 1 An assignment $\mu \in M$ is efficient if $\sum_{i \in I} a_{i \mu(i)} \geq \sum_{i \in I} a_{i \eta(i)}$ for all assignments $\eta \in M$.

Definition 2 An allocation $(\mu, p) \in M \times R^{n}$ is envy-free if $u_{i}\left(p_{\mu(i)}\right) \geq u_{i}\left(p_{j}\right)$ for all $i \in I$ and $j \in J$.

Given an envy-free allocation $(\mu, p) \in M \times R^{n}$ we will refer to $p$ as an envyfree price. Also denote by $M^{A}$ the set of efficient assignments relative to the matrix of valuations $A$. Alkan et al. [2] prove that if the allocation $(\mu, p)$ is envy-free then the assignment $\mu$ is efficient. One can also think of envy-freeness as a sufficient requirement of stability since each individual prefers his object to any other object given the vector of prices. Therefore envy-freeness is used as a solution concept in most models dealing with indivisible objects, see for example Alkan et al. [2], Aragonés [3], Haake et al. [8] and Klijn [9].

The assignment problem of indivisible objects was first addressed by Shapley and Shubik [13] who proved that the problem can be translated into a linear programming problem where the efficient assignments are obtained from the primal problem but envy-free prices and the corresponding utilities come from the dual problem as shadow prices. Given the matrix of valuations $A$, define with the coalitional function $w(A, T, Q)$ the maximal worth that a coalition of agents $T \subseteq I$ can obtain when assigned to a set of objects $Q \subseteq J$. It can be expressed in terms of the following linear programming problem ${ }^{2}$ : given the matrix of valuations $A$ and subsets $T$ and $Q$, choose $\left(x_{i j}\right)_{i \in T, j \in Q}$ to solve for

$$
w(A, T, Q) \equiv \max \sum_{i \in T, j \in Q} a_{i j} x_{i j}
$$

\footnotetext{
${ }^{1}$ See the discussion in Section 6 when the number of agents and objects is different. Note, however, that the results stated in this section do not require that $|I| \neq|J|$.

${ }^{2}$ The last condition is needed to ensure that agents will be assigned to objects even if their valuations are negative.
} 
subject to

$$
\begin{array}{cl}
\sum_{i \in T} x_{i j} \leq 1 & \text { for any } j \in Q \\
\sum_{j \in Q} x_{i j} \leq 1 & \text { for any } i \in T \\
x_{i j} \geq 0 & \text { for any } i \in T, j \in Q \\
\sum_{i \in T, j \in Q} x_{i j}= & \min (|T|,|S|) .
\end{array}
$$

This primal problem has a corresponding dual problem where the costs of inputs - agents and objects - are minimized. Shadow prices are prices of objects and utilities of agents. Given the matrix of valuations $A$ and subsets $T$ and $Q$, choose $\left(u_{i}\right)_{i \in T}$ and $\left(p_{j}\right)_{j \in Q}$ to solve for

$$
w(A, T, Q) \equiv \min \sum_{i \in T} u_{i}+\sum_{j \in Q} p_{j}
$$

subject to

$$
u_{i}+p_{j} \geq a_{i j} \quad \text { for any } i \in T, j \in Q
$$

Then the solution of the primal has the property that $x_{i j}$ takes values 0 or 1 for all $i \in T$ and all $j \in Q$. Assume that $T=I$ and $Q=J$. The primal problem solves for an efficient assignment of objects as follows: given the solution $\left(x_{i j}\right)_{i \in I, j \in J}$, define the assignment $\mu$ by letting $\mu(i)=j$ if and only if $x_{i j}=1$. The dual problem gives the set of envy-free prices (this follows from constraint (3) since $u_{i} \geq a_{i j}-p_{j}$ for all $i \in I$ and all $j \in J$ ) and the corresponding utilities $\left(u_{i} \equiv u_{i}\left(p_{\mu(i)}\right)\right)$. The set of envy-free prices forms a lattice that possess the following property: if $p^{\prime}$ and $p^{\prime \prime}$ are two envy-free price vectors then so are the price vectors $\underline{p}$ and $\bar{p}$ where $\underline{p}_{i}=\min \left(p_{i}^{\prime}, p_{i}^{\prime \prime}\right)$ and $\bar{p}_{i}=\max \left(p_{i}^{\prime}, p_{i}^{\prime \prime}\right)$. This property is proven in Shapley and Shubik [13], see also Roth and Sotomayor [12] (chapter 8). The lattice has an agent-optimal price vector $p_{*} \geq 0$ such that $p \geq p_{*} \geq 0$ for all envy-free and non-negative prices $p$.

Given an efficient assignment $\mu$, an agent-optimal price can be calculated ${ }^{3}$ (see Leonard [10] or Roth and Sotomayor [12]) using the coalitional function, defined by equation (1), as

$$
p_{* \mu(i)}=w(A, I \backslash\{i\}, J)-w(A, I \backslash\{i\}, J \backslash\{\mu(i)\}),
$$

for each $i \in I$. From (4) it follows that $p_{* \mu(i)}$ does not depend on the object valuations of agent $i$. Using this property Leonard [10] proves that the mechanism that selects the agent-optimal prices $p_{*}$ is strategy-proof. His result is a

\footnotetext{
${ }^{3}$ For an example how to calculate agent-optimal prices, see Section 4.
} 
consequence of the well-known Clark's pivotal mechanism and is a special case of the results proven by Roberts [11] for quasilinear utility functions.

Here I state some additional results that will be useful later in proving the theorem. ${ }^{4}$

Proposition 1 Given a matrix of valuations $A$, the coalitional function $w(A, T, Q)$ is continuous and weakly increasing in $a_{i j}$.

It is shown in the proof of the Proposition 1 that when $i \in T$ and $j \in Q$ equation (1) can be written as

$$
w(A, T, Q)=\max \left(\text { const }_{1}+a_{i j} \cdot 0, \text { const }_{2}+a_{i j} \cdot 1\right)
$$

where const $_{2}=w(A, T \backslash\{i\}, Q \backslash\{j\})$ and const $_{1}$ is a coalitional worth that is obtained by solving the original assignment problem subject to the additional constraint that agent $i$ is not assigned to object $j$. Since neither const $_{1}$ nor const $_{2}$ is affected by the change in $a_{i j}$ they can be considered constants. Function (5) is obviously continuous and weakly increasing in $a_{i j}$.

Proposition 2 Given a matrix of valuations $A$, the set of envy-free prices is the same for all efficient assignments of objects.

Proposition 2 allows us to establish immediately the following result.

Corollary 1 Fix an envy-free price vector $p$. Under all efficient assignments of objects each agent gets the same utility:

$$
a_{i \mu_{1}(i)}-p_{\mu_{1}(i)}=a_{i \mu_{2}(i)}-p_{\mu_{2}(i)}
$$

for all $i \in I$ and where $\mu_{1}$ and $\mu_{2}$ are any two efficient assignments of objects.

\section{Implementation Problem}

In the implementation problem that I consider I restrict the set of feasible price vectors and require the prices to sum to a given number $C: \sum_{j \in J} p_{j}=C$. One can think in terms of an economy that consists of the set $J$ of indivisible objects and the divisible object - money - of quantity $C$ that must be assigned among $n$ agents.

Let $\triangle_{C}$ denote the set of price vectors that sum to $C$. Since all feasible price vectors are required to belong to this set $p \in \triangle_{C}$, from now on it is understood that an allocation is an assignment-price pair $(\mu, p) \in M \times \triangle_{C}$. And with an envy-free price vector $p$ I will only refer to price vectors that meet the price constraint: $p \in \triangle_{C}$.

\footnotetext{
${ }^{4}$ The proofs are provided in the Appendix.
} 
Given $C$ and the matrix of valuations $A$, denote the set of envy-free allocations in $M \times \triangle_{C}$ with $G(A)$. If a social planner were to choose an allocation $(\mu, p)$, arguably, he would prefer to select one from the set of envy-free allocations $(\mu, p) \in G(A)$ since these allocations meet the desirable normative criteria of envy-freeness and hence efficiency. The algorithms proposed by Abdulkadiroğlu et al. [1], Aragonés [3], Brams and Kilgour [5], Haake et al. [8], and Klijn [9] were designed to select allocations from the set $G(A)$. However, all of them rely on the knowledge of matrix $A$. If the social planner does not know the true preferences of agents, he will need to solicit them. A question arises whether agents have strategic incentives to reveal their true valuations. That is, an agent can find it profitable to announce valuations of objects different from his true ones. ${ }^{5}$ Given this misrepresentation of preferences there is no guarantee anymore that the selected allocation by any of the algorithms will satisfy envy-freeness with respect to the true preferences. However, it will be demonstrated that, with the help of an appropriate tie-breaking rule, selecting an allocation that is envy-free with respect to the announced preferences, not necessarily the true ones, achieves envy-freeness with respect to the true valuations in the equilibrium.

Formally, a strategy of agent $i$ is a vector of real-valued valuations of objects $b_{i}=\left(b_{i 1}, \ldots, b_{i n}\right) \in R^{n}$ that he announces. Given the matrix of reported valuations $B=\left[b_{i j}\right]_{i \in I, j \in J}$, denote the set of envy-free allocations implied by the matrix $B$ by $G(B)$. A mechanism $g$ is a mapping from the space of valuations into the space of allocations $g: R^{n \times n} \rightarrow M \times \triangle_{C}$. I restrict attention to mechanisms that, for each matrix of valuations $B$, will select an allocation $(\mu, p)$ from the envy-free set $G(B)$. The justification why to consider such mechanisms was provided before - there already exist algorithms that select allocations that are envy-free with respect to a given matrix of valuations.

In general, the set of envy-free prices is not a singleton. From all envy-free prices the price vector that I select corresponds to the one that would be selected according to the algorithm of Abdulkadiroğlu et al. [1] when applied to the matrix $B$. The advantage of this price vector is its explicit linear relationship with the agent-optimal prices, given by the equation:

$$
p_{j}=p_{* j}+\frac{C-\sum_{m \in J} p_{* m}}{n} \text { for all } j \in J .
$$

where $p_{*}$ is the vector of agent-optimal prices implied by $B$. According to this formula each agent $i \in I$ pays the agent-optimal price corresponding to the object he gets $p_{* \mu(i)}$ plus the equal share of the difference between the price constraint and the sum of all agent-optimal prices.

\footnotetext{
${ }^{5}$ Truth-telling is a dominant strategy when the assignment $\mu$ is efficient and agent $i$ pays the agent-optimal price $p_{* \mu(i)}$ of the object he gets. However, in general the agent-optimal prices will not meet the price constraint, $p_{*} \notin \triangle_{C}$, and therefore, $\left(\mu, p_{*}\right) \notin G(A)$.
} 
The utility of agent $i$ having object $\mu(i)$ and paying price $p_{\mu(i)}$, by applying equation (6), is

$$
u_{i}\left(p_{\mu(i)}\right)=a_{i \mu(i)}-p_{\mu(i)}=a_{i \mu(i)}-\frac{C}{n}-\frac{n-1}{n} p_{* \mu(i)}+\frac{1}{n} \sum_{l \neq i} p_{* \mu(l)} .
$$

It follows that the utility of agent $i$ is decreasing in its own agent-optimal price but increasing in each of other agent-optimal prices keeping the assignment $\mu$ fixed. We know from equation (4) that $p_{* \mu(i)}$ does not depend on the valuations of objects reported by agent $i$, that is, he cannot affect his own agent-optimal price. However, he can affect the agent-optimal prices of other objects.

If there are several efficient assignments of agents to objects with respect to the reported valuations $B$ then the mechanism $g$ will break ties according to the following rule. Order all objects and all agents, and without loss of generality assume that the order corresponds to the natural one: $\sigma(i)=i$ for all $i \in I$ and $\sigma(j)=j$ for all $j \in J$, and keep these orders fixed. Start with object 1 and proceed iteratively. If all efficient assignments allocate object 1 to the same agent $i$, then let agent $i$ get it. Otherwise choose among all efficient assignments the one that assigns object 1 to the agent that has announced the smallest valuation for object 1: $\mu(i)=1$ if $b_{i 1}<b_{k 1}$ for any $k$ such that there exists an efficient assignment $\nu \in M^{B}$ under which $\nu(k)=1$. If $b_{i 1}=b_{k 1}$ for two or more agents then select the agent from this set who has been assigned the lowest number: $\mu(i)=1$ if $i<k$ when $b_{i 1}=b_{k 1}$. In general, assume that objects 1 to $l-1$ are already assigned. If all remaining efficient assignments allocate object $l$ to the same agent $i$, then let agent $i$ get it. Otherwise choose among all efficient assignments the one that assigns object $l$ to the agent that has announced the smallest valuation of object $l: \mu(i)=l$ if $b_{i l}<b_{k l}$ for any $k$ such that there exists an efficient assignment $\nu \in M^{B}$ such that $\nu(k)=l$ and $\nu^{-1}(j)=\mu^{-1}(j)$ for already assigned objects $j \in\{1, \ldots, l-1\}$. If $b_{i l}=b_{k l}$ for two or more agents select the agent from this set who has been assigned the lowest number: $\mu(i)=l$ if $i<k$ when $b_{i l}=b_{k l}$. Thus the tie-breaking rule selects a unique assignment among all efficient assignments with respect to $B$. Thus, the mechanism $g$ defines a game form, and given $A$, the pair $(A, g)$ is a game in normal form.

Assume that the strategy profile $B$ has been announced. When a set of agents $T$ deviates and announces a different vector of valuations $b_{T}^{\prime} \in R^{|T| \times n}$, that leads to another profile $B^{\prime}=\left(b_{T}^{\prime}, b_{-T}\right)$. When there is only one deviator, $T=\{i\}$, the strategy profile after the deviation is denoted by $B^{\prime}=\left(b_{i}^{\prime}, b_{-i}\right)$. Denote the allocation induced by the deviation by $g\left(B^{\prime}\right)=\left(\mu^{\prime}, p^{\prime}\right)$. The solution concept that I use is strong Nash equilibrium.

Definition $3 A$ strategy profile $B \in R^{n \times n}$ is a strong Nash equilibrium relative to $(A, g)$ if there is no coalition $T$ and strategy profile $b_{T}^{\prime}$ such that $u_{i}\left(p_{\mu^{\prime}(i)}^{\prime}\right) \geq u_{i}\left(p_{\mu(i)}\right)$ for all $i \in T$ and $u_{i}\left(p_{\mu^{\prime}(i)}^{\prime}\right)>u_{i}\left(p_{\mu(i)}\right)$ for at least one $i \in T$. 
Denote the set of strong Nash equilibrium outcomes relative to $(A, g)$ by $O_{(A, g)}^{S N E}$, that is $O_{(A, g)}^{S N E}=\left\{(\mu, p) \in M \times \triangle_{C} \mid g(B)=(\mu, p)\right.$ for some pure strategy strong Nash equilibrium $B$ relative to $(A, g)\}$. Similarly, we can define Nash equilibrium if we restrict the set of deviators $T$ to a single agent $i$ and denote the set of Nash equilibrium outcomes relative to $(A, g)$ by $O_{(A, g)}^{N E}$.

Now we are ready to state the main result of the paper:

Theorem The mechanism $g$ implements the social choice correspondence $G$ in strong Nash equilibrium: $O_{(A, g)}^{S N E}=G(A)$ for all $A \in R^{n \times n}$.

\section{Example}

Before providing the proof of the theorem, consider the following numeric two agent-two object example with the price constraint $C=20$ and the matrix of valuations

$$
A=\left(\begin{array}{cc}
15 & 18 \\
6 & 22
\end{array}\right)
$$

The efficient assignment is $\mu(1)=1$ and $\mu(2)=2$ since $15+22>6+18$. The set of all envy-free prices is delimited by the equations $p_{2}=3+p_{1}$ and $p_{2}=16+p_{1}$ and shown in Figure 1 by the shaded area. To obtain the agent-optimal price of object 1 , we find that $w(A, I \backslash\{1\}, J)=22$ and $w(A, I \backslash\{1\}, J \backslash\{\mu(1)\})=22$, and by applying equation (4), $p_{* 1}=22-22=0$. In the same way we can find that $p_{* 2}=18-15=3$. Thus the agent-optimal prices are $p_{*}=(0,3)$.

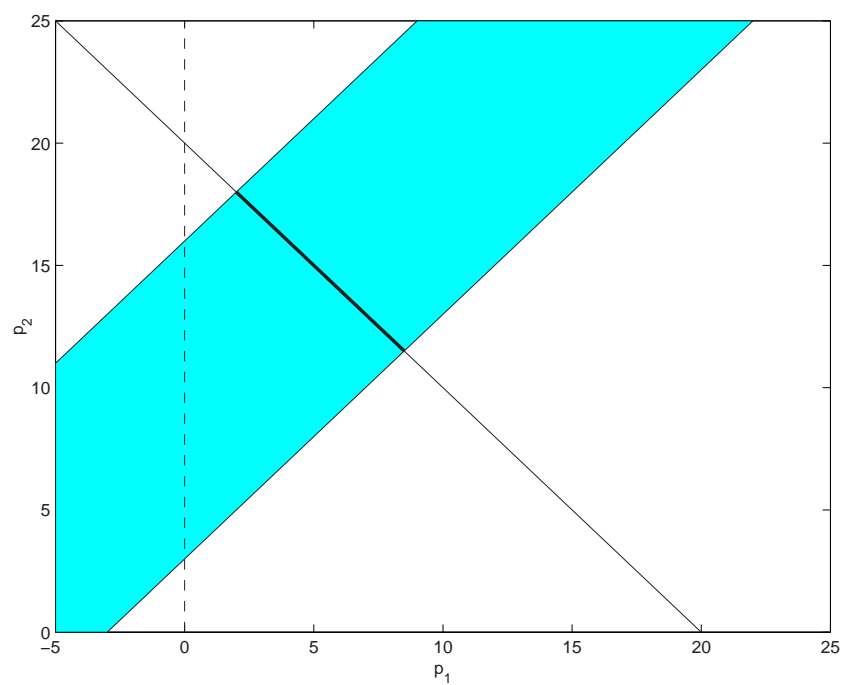

Figure 1: The set of envy-free prices 
The prices that sum up to $C$ are represented with the line connecting the points $(20,0)$ and $(0,20)$. The intersection of this line with the shaded region gives the set of envy-free prices that meet the price constraint. In general, there are an infinity of prices that are envy-free and meet the price constraint. The mechanism $g$ that I consider selects, given the announced valuations, envy-free prices obtained from the agent-optimal prices by increasing all of them by the same amount so that the price constraint is met. If the announced valuations are $A$, then the vector of prices selected by the mechanism is $p=(8.5,11.5)$ (found by adding 8.5 to the agent-optimal prices $p_{*}$ ).

The algorithm by Abdulkadiroğlu et al. [1] would also select the prices $p=$ $(8.5,11.5)$. Their algorithm finds the first envy-free price when we move from the initial price vector $p^{0}=\frac{C}{n}$ along the rent constraint. Thus, the price $p$ obtained by the algorithm is the most 'equal' price among all envy-free prices. In the example we start from $p^{0}=(10,10)$ and reach $p=(8.5,11.5)$. The proposed mechanism does not ensure neither nonnegative prices nor individual rationality since it depends on the magnitude of $C{ }^{6}$ In the example, if $C<3$ all envy-free price vectors have at least one price negative and if $C>37$ then there is no envy-free price that would be individually rational.

In general, agents do not have incentives to announce the true valuations. Agent 1 by announcing the vector $b=(2,18)$ still gets object 1 according to the tie-breaking rule but pays 2 instead of 8.5. Since in order to find an efficient assignment what matters is the relative magnitudes of valuations we can define $\beta_{i} \equiv b_{i 2}-b_{i 1}$. Then agent $i$ gets object 2 and agent $k$ gets object 1 if $\beta_{i}>\beta_{k}$ or if $\beta_{i}=\beta_{k}$ and $b_{i 1}>b_{k 1}$. One can check that when agent $i$ gets object 2 and agent $k$ gets object 1 the agent-optimal prices are given by $p_{* 1}=\max \left(-\beta_{i}, 0\right)$ and $p_{* 2}=\max \left(\beta_{k}, 0\right)$. It is easy to check that any strategy profile $B$ where $3 \leq \beta_{1}=$ $\beta_{2} \leq 16$ and $b_{11} \leq b_{21}$ is a Nash equilibrium. When $\beta_{1}=\beta_{2}$ both assignments are efficient with respect to the announced preferences, but by announcing $b_{11} \leq b_{21}$ tie-breaking rule ensures that the mechanism will select the assignment that is also efficient with respect to the true preferences.

In the proof of the theorem I consider two types of deviations when an agent feels envy. The first occurs when the agent after the deviation still gets the same object but the other agent now must pay a higher price and thus, according to the price constraint, the deviating agent pays a lower price. For example, if $\beta_{2}>\beta_{1}, \beta_{2}>0$ and $\beta_{1}<3$ and matrix $A$ represents the true preferences. Given the announced preferences, $\mu(1)=1$ and $\mu(2)=2$ and the agent-optimal prices are $p_{* 1}=\max \left(-\beta_{2}, 0\right)=0$ and $p_{* 2}=\max \left(\beta_{1}, 0\right)$. Therefore $p_{1}=p_{* 1}+(20-$ $\left.p_{* 1}-p_{* 2}\right) / 2=\left(20-\max \left(\beta_{1}, 0\right)\right) / 2>8.5$ and $p_{2}=20-p_{1}<11.5$. Agent 1 feels envy since $15-p_{1}<18-p_{2}$. Agent 1 can deviate and announce $\beta_{1}^{\prime}=\beta_{2}$ and $b_{11}^{\prime}<b_{21}$. Then agent still gets object 1 but pays only $p_{1}^{\prime}=\left(20-\beta_{2}\right) / 2<p_{1}$ since $p_{* 1}^{\prime}=\max \left(-\beta_{2}, 0\right)=0$ and $p_{* 2}^{\prime}=\max \left(\beta_{1}^{\prime}, 0\right)=\beta_{2}$. Thus he had a profitable

\footnotetext{
${ }^{6}$ An allocation $(\mu, p) \in M \times \triangle_{C}$ is individually rational if $u_{i \mu(i)}\left(p_{\mu(i)}\right) \geq 0$ for all $i \in I$.
} 
deviation.

The second type of deviation occurs when an agent gets the object he envies at the price that the agent who was originally assigned to it paid. For example, if $0 \geq \beta_{2}>\beta_{1}$ and matrix $A$ represents the true preferences then $\mu(1)=1$ and $\mu(2)=2, p_{* 1}=\max \left(-\beta_{2}, 0\right)=-\beta_{2}$ and $p_{* 2}=\max \left(\beta_{1}, 0\right)=0$. Agent 1 must pay $p_{1}=\left(20-\beta_{2}\right) / 2>8.5$ and like in the previous case, feels envy. Agent 1 can profitably deviate by announcing $\beta_{1}^{\prime}=\beta_{2}$ and $b_{11}^{\prime}>b_{21}$. After the deviation the efficient assignment is $\mu^{\prime}(1)=2$ and $\mu^{\prime}(2)=1$ with agentoptimal prices $p_{* 1}^{\prime}=\max \left(-\beta_{1}^{\prime}, 0\right)=-\beta_{2}$ and $p_{* 2}^{\prime}=\max \left(\beta_{2}, 0\right)=0$. Agent 1 pays $p_{2}^{\prime}=p_{2}=\left(20+\beta_{2}\right) / 2<11.5$. A similar profitable deviation exists when $\beta_{2}=\beta_{1}$ and $b_{11}=b_{21}$. Then agent 1 is assigned to object 1 and will feel envy if $\beta_{1}<3$. Agent 1 is strictly better off by announcing $b_{11}^{\prime}>b_{21}$ while keeping $\beta_{1}^{\prime}=\beta_{2}$. If $\beta_{1}=3$ then agent 1 is indifferent between getting object 1 and 2 . Observe that examples discussed cover all the cases when agent 1 could feel envy when he is originally assigned to object 1 .

When there are more than two agents, it gets a little bit more complicated to demonstrate the existence of a profitable deviation when an agent feels envy. It may not be anymore possible either to increase the price paid by the agent who is assigned to the object that is envied or to obtain that object at the price that the agent who was originally assigned to it paid. For example, consider the following matrix of announced preferences

$$
B=\left(\begin{array}{ccc}
5 & 10 & 15 \\
5 & 10 & 0 \\
0 & 10 & 20
\end{array}\right)
$$

and $C=30$ then agent optimal prices are $p_{*}=(0,5,10)$ and prices selected that sum to 30 are $p=(5,10,15)$. There are two efficient assignments $\mu_{1}(1)=$ $1, \mu_{1}(2)=2, \mu_{1}(3)=3$ and $\mu_{2}(1)=2, \mu_{2}(2)=1, \mu_{2}(3)=3$. The tie-breaking rule selects the first assignment. Suppose that agent 3 envies object 2 at the given prices: $a_{32}-10>a_{33}-15$. Agent 3 can not increase the prices of objects 1 and/or 2 and thus decrease the price of object 3 and still get it. And neither he can obtain object 2 at price $p_{2}=10$. By announcing the vector of valuations $b_{3}^{\prime}=(0,15+\epsilon, 20)$ where $\epsilon>0$ ensures that $\mu^{\prime}(3)=2$ and the agent optimal prices will be $p_{*}^{\prime}=(0,5,10-\epsilon)$ and selected prices $p=(5+\epsilon / 3,10+\epsilon / 3,15-2 \epsilon / 3)$. For $\epsilon$ sufficiently small the agent 3 will find it advantageous to deviate since $a_{32}-10-\epsilon / 3>a_{33}-15$.

\section{Proof of Theorem}

Throughout the proof fix a matrix of true valuations $A$, and assume without loss of generality that the orders of agents and objects needed to define $g$ are both $1,2, \ldots, n$. 
To establish the statement of the theorem, one needs to demonstrate, first, that for every envy-free allocation one can construct a strategy profile $B$ that is a strong Nash equilibrium of the proposed game $(A, g)$ (Lemma 1$)$; second, that a strategy profile $B$ where an agent feels envy at allocation $g(B)=(\mu, p)$ can not be a strong Nash equilibrium of the game $(A, g)$ (Lemma 2 ).

Lemma 1 Let $(\mu, p)$ be an envy-free allocation. Then there is a strong Nash equilibrium $B$ of $(A, g)$ such that $g(B)=(\mu, p)$.

Proof: Take an envy-free allocation $(\mu, p) \in G(A)$. Consider the following strategy profile $B$ : each agent $i \in I$ announces $b_{i}=p+c_{i}$ and scalars $c_{i}$ have the following relationship for any two agents $i$ and $k: c_{i}<c_{k}$ if and only if $\mu(i)<\mu(k)$. I claim that the given strategy profile constitutes a strong Nash equilibrium.

Observe that any possible assignment of objects is efficient with respect to $B$. The only envy-free price vector is $p$. The way how the scalars $c_{i}$ for $i=\{1, \ldots, n\}$ were chosen ensures according to the tie-breaking rule that the unique assignment selected will be $\mu$ : an agent $i$ who announced the smallest $b_{i 1}$ among all agents will be assigned to object 1 and by construction it was agent $\mu^{-1}(1)$. Among the remaining $n-1$ agents, agent $\mu^{-1}(2)$ announced the smallest $b_{i 2}$ therefore he is assigned to object 2 and so forth.

Assume on the contrary that there exists a profitable deviation by a group of agents $T$. Given the strategy profile after deviation $B^{\prime}=\left(b_{T}^{\prime}, b_{-T}\right)$, the mechanism $g$ selects an allocation $\left(\nu, p^{\prime}\right)$. Since before deviation all agents $i \in T$ preferred their object to any other object and for a deviation to be profitable it must be that

$$
a_{i \nu(i)}-p_{\nu(i)}^{\prime} \geq a_{i \mu(i)}-p_{\mu(i)} \geq a_{i \nu(i)}-p_{\nu(i)}
$$

with the first inequality strict for at least one agent $i \in T$. It follows that for all $i \in T$

$$
p_{\nu(i)}^{\prime} \leq p_{\nu(i)}
$$

with at least one inequality strict. Thus there exits an object $j$ whose price has strictly decreased: $p_{j}^{\prime}<p_{j}$. Observe that if $T=I$ it follows immediately that the new price vector does not sum to $C$, a contradiction.

If $T \varsubsetneqq I$ choose the object $j$ whose price has decreased the most. Since after the deviation the selected allocation $g\left(B^{\prime}\right)=\left(\nu, p^{\prime}\right)$ is envy-free with respect to the matrix $B^{\prime}$ then for each non-deviating agent $i \in I \backslash T$ we have an inequality

$$
b_{i \nu(i)}-p_{\nu(i)}^{\prime} \geq b_{i j}-p_{j}^{\prime} .
$$

Using the fact that before the deviation $b_{i \nu(i)}-p_{\nu(i)}=b_{i j}-p_{j}$ since $b_{i}=p+c_{i}$ we obtain for each agent $i \in I \backslash T$ that

$$
0>p_{j}^{\prime}-p_{j} \geq p_{\nu(i)}^{\prime}-p_{\nu(i)}
$$


for the assignment $\nu$. Thus it follows that $p_{\nu(i)}^{\prime}<p_{\nu(i)}$ for all $i \in I \backslash T$. Combining it with (9) and summing up over all the agents gives

$$
\sum_{j=1}^{n} p_{j}^{\prime}<\sum_{j=1}^{n} p_{j}
$$

a contradiction since both price vectors must sum to $C$.

Lemma 1 says that an envy-free allocation can be supported as a strong Nash equilibrium of $(A, g)$. Therefore, if $(\mu, p) \in G(A)$ then $(\mu, p) \in O_{(A, g)}^{S N E}$. Note also that the proof does not depend on any particular way the prices are determined as long as they are envy-free with respect to the announced matrix $B$.

Lemma 2 Let $B$ be a strategy profile such that $g(B)=(\mu, p) \notin G(A)$. Then $B$ is not a strong Nash equilibrium of $(A, g)$.

Proof: Let $g(B)=(\mu, p)$ be given and assume that agent $i$ envies object $j$ :

$$
u_{i}\left(p_{j}\right)>u_{i}\left(p_{\mu(i)}\right)
$$

I will construct a profitable deviation in two steps. In the first step consider a possible deviation $b_{i}^{\prime}$ where agent $i$ announces

$$
b_{i j}^{\prime}=w(B, I, J)-w(B, I \backslash\{i\}, J \backslash\{j\}) \geq b_{i j}
$$

and $b_{i k}^{\prime}=b_{i k}$ for all $k \neq j$. According to (5) we can distinguish between two cases before the deviation. First, there was an efficient assignment $\nu \in M^{B}$ such that $\nu(i)=j$. Then we have const $_{1} \leq w(B, I \backslash\{i\}, J \backslash\{j\})+b_{i j}=w(B, I, J)$. Then by the construction of the deviation $b_{i j}^{\prime}=b_{i j}$ and $w\left(B^{\prime}, I, J\right)=w(B, I, J)$. Second, there was no efficient assignment $\nu \in M^{B}$ such that $\nu(i)=j$. It implies that $w(B, I \backslash\{i\}, J \backslash\{j\})+b_{i j}<$ const $_{1}=w(B, I, J)$. By substituting this result for const $_{1}$ in equation (5) but applied to calculate $w\left(B^{\prime}, I, J\right)$, it again follows that $w\left(B^{\prime}, I, J\right)=w(B, I, J)$. Since after the deviation every assignment that achieves the coalitional worth equal to $w\left(B^{\prime}, I, J\right)$ is efficient, it follows that all assignments that were efficient before the deviation remain efficient after. That is, the deviation $b_{i}^{\prime}$ was constructed in such a way that no assignment that was efficient is destroyed by the deviation and if the deviation adds an additional efficient assignment, it must assign agent $i$ to object $j$ : if $\nu \in M^{B}$ then $\nu \in M^{B^{\prime}}$, and if $\nu \in M^{B^{\prime}}$ but $\nu \notin M^{B}$ then $\nu(i)=j$. It follows that $\mu \in M^{B^{\prime}}$. Therefore we can take the assignment $\mu$ to find the agent-optimal price of any object $l \in J$ after the deviation according to (4):

$$
p_{* l}^{\prime}=w\left(B^{\prime}, I \backslash\left\{\mu^{-1}(l)\right\}, J\right)-w\left(B^{\prime}, I \backslash\left\{\mu^{-1}(l)\right\}, J \backslash\{l\}\right) .
$$


Since $w\left(B^{\prime}, I, J\right)=w(B, I, J)$ and $b_{\mu^{-1}(l) l}^{\prime}=b_{\mu^{-1}(l) l}$ for all $l \in J$ because the only valuation to change was $b_{i j}$ but $\mu(i) \neq j$, therefore the second term of (13) does not change:

$$
w\left(B^{\prime}, I \backslash\left\{\mu^{-1}(l)\right\}, J \backslash\{l\}\right)=w\left(B^{\prime}, I, J\right)-b_{\mu^{-1}(l) l}^{\prime}=w(B, I, J)-b_{\mu^{-1}(l) l} .
$$

By Proposition 1 the first term is weakly increasing in $b_{i j}$ :

$$
w\left(B^{\prime}, I \backslash\left\{\mu^{-1}(l)\right\}, J\right) \geq w\left(B, I \backslash\left\{\mu^{-1}(l)\right\}, J\right) .
$$

Therefore none of the agent-optimal prices can decrease as a result of the deviation.

In the continuation I analyze the following two cases:

Case 1 The agent-optimal price of object $j$ strictly increases: $p_{* j}^{\prime}>p_{* j}$.

It means that

$$
w\left(B^{\prime}, I \backslash\left\{\mu^{-1}(j)\right\}, J\right)>w\left(B, I \backslash\left\{\mu^{-1}(j)\right\}, J\right) .
$$

It can only happen if $b_{i j}^{\prime}>b_{i j}$, which means that there was no efficient assignment $\nu \in M^{B}$ that would allocate agent $i$ to object $j$. Applying (5) we obtain that ${ }^{7}$

$$
\begin{aligned}
w\left(B^{\prime}, I \backslash\left\{\mu^{-1}(j)\right\}, J\right) & =\text { const }_{2}+b_{i j}^{\prime}> \\
w\left(B, I \backslash\left\{\mu^{-1}(j)\right\}, J\right) & =\max \left(\text { const }_{1}, \text { const }_{2}+b_{i j}\right) .
\end{aligned}
$$

Now consider a deviation where agent $i$ announces, given a sufficiently small $\epsilon>0$

$$
b_{i j}^{\prime \prime}=b_{i j}^{\prime}-\epsilon>b_{i j}
$$

and $b_{i k}^{\prime \prime}=b_{i k}$ for all $k \neq j$. In what follows I compare the strategy profile after the deviation $B^{\prime \prime}=\left(b_{i}^{\prime \prime}, b_{-i}\right)$ with the initial strategy profile $B=\left(b_{i}, b_{-i}\right)$. First, after the deviation the set of efficient assignments does not change $M^{B}=M^{B^{\prime \prime}}$, and so does the selected assignment: $\mu \in M^{B}$. Second, using the same argument as when discussing the deviation $B^{\prime}=\left(b_{i}^{\prime}, b_{-i}\right)$, none of agent-optimal prices can decrease as a result of the deviation. Third, from (14) it follows that

$$
w\left(B^{\prime}, I \backslash\left\{\mu^{-1}(j)\right\}, J\right)>w\left(B^{\prime \prime}, I \backslash\left\{\mu^{-1}(j)\right\}, J\right)>w\left(B, I \backslash\left\{\mu^{-1}(j)\right\}, J\right),
$$

and as a result $p_{* j}^{\prime \prime}=p_{* j}^{\prime}-\epsilon>p_{* j}$. Fourth, the agent-optimal price of object $\mu(i)$ does not change $p_{\mu(i)}=p_{\mu(i)}^{\prime \prime}$ since by (4) it does not depend on the valuations of agent $i$. Then, according to (7), agent $i$ is strictly better off after the deviation $b_{i}^{\prime \prime}$, thus strategy profile $B$ was not an equilibrium.

\footnotetext{
${ }^{7}$ Observe that the values of constants const $_{1}$ and const $_{2}$ change depending on the sets $T \subseteq I$ and $Q \subseteq J$ but not on the value of $b_{i j}$ while keeping the rest $b_{k l}$, for $k \neq i$ and $l \neq j$, fixed.
} 
Case 2 The agent-optimal price of object $j$ remains the same: $p_{* j}^{\prime}=p_{* j}$.

First I argue that this case implies that none of the agent-optimal prices will change due to the deviation $b_{i}^{\prime}$, namely, $p_{* k}^{\prime}=p_{* k}$ for all $k \in J$. From Proposition 2 , in order to check whether a price vector is envy-free, it is sufficient to consider any efficient assignment. Choose $\mu \in M^{B}$ since by the construction of $B^{\prime}$ the assignment $\mu \in M^{B^{\prime}}$. Clearly, the only agent who could feel envy under the price vector $p_{*}$, given the matrix of valuations $B^{\prime}$, is agent $i$ and only with respect to object $j$, that is, $b_{i \mu(i)}-p_{* \mu(i)}<b_{i j}^{\prime}-p_{* j}$. However, the deviation was constructed to ensure that there exists an assignment $\nu \in M^{B^{\prime}}$ such that $\nu(i)=j$. Corollary 1 says that $b_{i \mu(i)}-p_{* \mu(i)}^{\prime}=b_{i j}^{\prime}-p_{* j}$ but agent $i$ cannot affect the agent-optimal price of the object he is assigned to under some efficient assignment rule therefore $p_{* \mu(i)}^{\prime}=p_{* \mu(i)}$. Thus nobody feels envy relative to $B^{\prime}$ under price vector $p_{*}$. And it was argued before that as a result of the deviation $b_{i}^{\prime}$, the agent-optimal prices cannot decrease, therefore $p_{*}$ must be vector of agent-optimal prices after the deviation.

Now consider a deviation $b_{i}^{\prime \prime}$ where agent $i$ announces, for sufficiently small $\epsilon$,

$$
b_{i j}^{\prime \prime}=b_{i j}^{\prime}+\epsilon
$$

and $b_{i k}^{\prime \prime}=b_{i k}$ for all $k \neq j$. After the deviation all efficient assignments will allocate object $j$ to agent $i: \nu(i)=j$ for all $\nu \in M^{B^{\prime \prime}}$ and $B^{\prime \prime}=\left(b_{i}^{\prime \prime}, b_{-i}\right)$. In what follows I compare the situation when the strategy profile $B^{\prime}=\left(b_{i}^{\prime}, b_{-i}\right)$ was used with the strategy profile $B^{\prime \prime}=\left(b_{i}^{\prime \prime}, b_{-i}\right)$. Take any efficient assignment after the deviation $b_{i}^{\prime \prime}: \nu \in M^{B^{\prime \prime}}$. This assignment was efficient before the deviation: $\nu \in M^{B^{\prime}}$. Again, agent $i$ cannot affect his own agent-optimal price, here, the price of object $j$. According to (4), before the deviation the price of any object $l \neq j$ is equal to

$$
p_{* l}=w\left(B^{\prime}, I \backslash\left\{\nu^{-1}(l)\right\}, J\right)-w\left(B^{\prime}, I \backslash\left\{\nu^{-1}(l)\right\}, J \backslash\{l\}\right)
$$

where

$$
w\left(B^{\prime}, I \backslash\left\{\nu^{-1}(l)\right\}, J \backslash\{l\}\right)=w\left(B^{\prime}, I \backslash\left\{\nu^{-1}(l), i\right\}, J \backslash\{l, j\}\right)+b_{i j}^{\prime}
$$

since $\nu(i)=j$. After the deviation $b_{i j}^{\prime \prime}$ the second term of (15) has increased by $\epsilon$, that is,

$$
w\left(B^{\prime \prime}, I \backslash\left\{\nu^{-1}(l)\right\}, J \backslash\{l\}\right)=w\left(B^{\prime}, I \backslash\left\{\nu^{-1}(l)\right\}, J \backslash\{l\}\right)+\epsilon
$$

for all $l \neq j$. The first term of (15) before the deviation is

$$
w\left(B^{\prime}, I \backslash\left\{\nu^{-1}(l)\right\}, J\right)=\max \left(\text { const }_{1}, \text { const }_{2}+b_{i j}^{\prime}\right) .
$$

Therefore, after the deviation $b_{i j}^{\prime \prime}$, it belongs to the interval:

$$
w\left(B^{\prime}, I \backslash\left\{\nu^{-1}(l)\right\}, J\right)+\epsilon \geq w\left(B^{\prime \prime}, I \backslash\left\{\nu^{-1}(l)\right\}, J\right) \geq w\left(B^{\prime}, I \backslash\left\{\nu^{-1}(l)\right\}, J\right) .
$$


It follows that the agent-optimal prices of objects other than $j$ cannot increase and each of them can decrease at most by $\epsilon: p_{* l}-\epsilon \leq p_{* l}^{\prime \prime} \leq p_{* l}$ for all $l \neq j$. Since, according to (7), the utility of agent $i$ is increasing in the agent-optimal prices paid by other agents, consider the worst case: $p_{* l}^{\prime \prime}=p_{* l}-\epsilon$ for all $l \neq j$. Then the utility of the agent $i$ after the deviation is $u_{i}\left(p_{j}^{\prime \prime}\right)=u_{i}\left(p_{j}\right)-\frac{n-1}{n} \epsilon$. By (12), for sufficiently small $\epsilon$,

$$
u_{i}\left(p_{j}^{\prime \prime}\right)=u_{i}\left(p_{j}\right)-\frac{n-1}{n} \epsilon>u_{i}\left(p_{\mu(i)}\right) .
$$

Thus, for sufficiently small $\epsilon$, announcing

$$
b_{i j}^{\prime \prime}=w(B, I, J)-w(B, I \backslash\{i\}, J \backslash\{j\})+\epsilon
$$

is a profitable deviation for agent $i$ and the matrix $B$ could not form a profile of Nash equilibrium strategies.

From Lemma 2 it follows that if $B$ is a Nash equilibrium of $(A, g)$ it must be envy-free, that is, if $g(B) \in O_{(A, g)}^{N E}$ then $g(B) \in G(A)$. Since $O_{(A, g)}^{S N E} \subseteq O_{(A, g)}^{N E}$ then the set of strong Nash equilibria belongs to the set of envy-free allocations $O_{(A, g)}^{S N E} \subseteq G(A)$. Lemma 1 already established the converse inclusion $G(A) \subseteq$ $O_{(A, g)}^{S N E}$. Therefore $G(A)=O_{(A, g)}^{S N E}$.

Since Lemma 1 implies that $G(A) \subseteq O_{(A, g)}^{S N E}$ and Lemma 2 implies that $O_{(A, g)}^{S N E} \subseteq$ $O_{(A, g)}^{N E} \subseteq G(A)$ we have an additional results.

Corollary 2 The sets of Nash equilibria and strong Nash equilibria of $(A, g)$ coincide: $O_{(A, g)}^{N E}=O_{(A, g)}^{S N E}$.

Corollary 3 The mechanism $g$ implements the social choice correspondence $G$ in Nash equilibrium: $O_{(A, g)}^{N E}=G(A)$ for all $A \in R^{n \times n}$.

\section{Concluding Remarks}

The model explicitly assumes that the number of agents and objects is the same. If the number of agents exceeded the number of objects one could introduce fictitious objects and the previous analysis would still apply. However, when the number of objects exceeds the number of agents, the introduction of fictitious agents does not work since it implies that fictitious agent will need to pay a price or receive a transfer of the object he is assigned to. As a result the actually paid prices would not meet the price constraint.

The feature of the mechanism is that an equilibrium strategy profile $B$ will usually imply multiple efficient assignments with respect to announced valuations. Therefore the mechanism always needs to rely on the tie-breaking rule to 
select the right assignment. I view this dependence on the tie-breaking rule as a drawback since the initial objective to select an envy-free allocation given the announced preferences has been coupled with a non-intuitive tie-breaking rule. One could substitute the present tie-breaking rule with another one where agents additionally to their valuations announce the object they prefer. However, my objective was to restrict the strategy space of agents to the announcements about their own valuations.

Observe that the mechanism does not ensure individual rationality. Thus it implicitly assumes that agents are forced to participate in the game although they may prefer to stay away. The set-up of the model imposes an exogenous price constraint. If $C$ is big enough then there will be no individually rational and envy-free allocations. However, if $C$ were not fixed, one could simply apply the results of Leonard [10] and Demange et al. [7] to implement in dominant strategies.

\section{Appendix: The Proofs of Propositions}

Proposition 1 Given a matrix of valuations $A$, the coalitional function $w(A, T, Q)$ is continuous and weakly increasing in $a_{i j}$.

Proof: If either $i \notin T$ or $j \notin Q$ then $w(A, T, Q)$ does not depend on $a_{i j}$ and can be treated as constant - obviously continuous and weakly increasing in $a_{i j}$. Assume that $i \in T$ and $j \in Q$. Given a solution $\left(x_{i j}\right)_{(i, j) \in T \times Q}$ to the primal problem, we can write equation (1) in the following form:

$$
w(A, T, Q)=\sum_{(k, l) \in T \times Q \backslash\{(i, j)\}} a_{k l} x_{k l}+a_{i j} x_{i j} .
$$

First, I claim that if as a result of the change from $a_{i j}$ to $a_{i j}^{\prime}$, keeping the rest of valuations fixed, there is no change in $x_{i j}=x_{i j}^{\prime}$, then there is no change in the solution $x_{k l}=x_{k l}^{\prime}$ for all $(k, l) \in T \times Q$. Assume, on the contrary, that $x_{k l} \neq x_{k l}^{\prime}$ for some $(k, l) \in T \times Q \backslash\{(i, j)\}$, and that $x_{k l}^{\prime}$ for all $(k, l) \in T \times Q$ was not another solution of the original problem. Then we can write the system of equations:

$$
\begin{aligned}
\sum_{(k, l) \in T \times Q \backslash\{(i, j)\}} a_{k l} x_{k l}^{\prime}+a_{i j} x_{i j} & <\sum_{(k, l) \in T \times Q \backslash\{(i, j)\}} a_{k l} x_{k l}+a_{i j} x_{i j} \\
\sum_{(k, l) \in T \times Q \backslash\{(i, j)\}} a_{k l} x_{k l}^{\prime}+a_{i j}^{\prime} x_{i j} & \geq \sum_{(k, l) \in T \times Q \backslash\{(i, j)\}} a_{k l} x_{k l}+a_{i j}^{\prime} x_{i j},
\end{aligned}
$$

where the first inequality holds under original valuations and the second holds after the change in $a_{i j}$. Thus we obtain a contradiction. Given this result, we can write equation (1) as

$$
w(A, T, Q)=\max \left(\text { const }_{1}+a_{i j} \cdot 0, \text { const }_{2}+a_{i j} \cdot 1\right) .
$$

The function in (17) is obviously continuous and weakly increasing in $a_{i j}$. Note that const $_{2}=$ $w(A, T \backslash\{i\}, Q \backslash\{j\})$ since agent $i$ has been assigned to object $j$ and each agent can be assigned to at most one object and vice versa.

Proposition 2 Given a matrix of valuations A, the set of envy-free prices is the same for all efficient assignments of objects. 
Proof: Take any two efficient assignments $\mu_{1}$ and $\mu_{2}$. Assume, on the contrary, that the price vector $p$ is envy-free for the assignment $\mu_{1}$ but it is not envy-free for the assignment $\mu_{2}$. Envy-freeness of $\mu_{1}$ implies that

$$
a_{i \mu_{1}(i)}-p_{\mu_{1}(i)} \geq a_{i \mu_{2}(i)}-p_{\mu_{2}(i)}
$$

for all $i \in I$. Assume without loss of generality that agent 1 envies object $j$ under assignment $\mu_{2}$ :

$$
a_{1 \mu_{1}(1)}-p_{\mu_{1}(1)} \geq a_{i j}-p_{j}>a_{1 \mu_{2}(1)}-p_{\mu_{2}(1)} .
$$

Summing up equation (18) across all agents and using equation (19) we obtain

$$
\sum_{i \in I} a_{i \mu_{1}(i)}-\sum_{i \in I} p_{\mu_{1}(i)}>\sum_{i \in I} a_{i \mu_{2}(i)}-\sum_{i \in I} p_{\mu_{2}(i)},
$$

contradicting the assumption that $\mu_{2}$ was an efficient assignment.

\section{References}

[1] A. Abdulkadiroğlu, T. Sönmez, and M.U. Ünver. Room assignment - rent division: A market approach. Social Choice and Welfare, 22:515-538, 2004.

[2] A. Alkan, G. Demange, and D. Gale. Fair allocation of indivisible objects and criteria of justice. Econometrica, 59:1023-1039, 1991.

[3] E. Aragonés. A derivation of the money rawlsian solution. Social Choice and Welfare, 12:267-276, 1995.

[4] C. Beviá. Manipulation games in economies with indivisible goods. May 2001.

[5] S.J. Brams and D.M. Kilgour. Competitive fair division. Journal of Political Economy, 109:418-443, 2001.

[6] S. Chin Sung and M. Vlach. Competitive envy-free division. Social Choice and Welfare, 23:103-111, 2004.

[7] G. Demange, D. Gale, and M. Sotomayor. Multi-item auctions. Journal of Political Economy, 94:863-872, 1986.

[8] C.-J. Haake, M.G. Raith, and F.E. Su. Bidding for envy-freeness: A procedural approach to $n$-player fair-division problems. Social Choice and Welfare, 17:201-215, 2002.

[9] F. Klijn. An algorithm for envy-free allocations in an economy with indivisible objects and money. Social Choice and Welfare, 17:201-215, 2000.

[10] H.B. Leonard. Elicitation of honest preferences for the assignment of individuals to positions. Journal of Political Economy, 91:461-479, 1983. 
[11] K. Roberts. The characterization of implementable choice rules. In Laffont J.J., editor, Aggregation and Revelation of Preferences, chapter 18, pages 321-349. North-Holland, Amsterdam, 1979.

[12] A.E. Roth and M.A. Oliveira Sotomayor. Two-sided matching: A study in game-theoretic modeling and analysis. Cambridge University Press, 1990.

[13] L. S. Shapley and M. Shubik. The assignment game 1: The core. International Journal of Game Theory, 1:111-130, 1972.

[14] K. Tadenuma and W. Thomson. Games of fair division. Games and Economic Behavior, 9:191-204, 1995. 